\title{
Plasmid-based Stat3-specific siRNA and GRIM-19 inhibit the growth of thyroid cancer cells in vitro and in vivo
}

\author{
GUI-MIN WANG ${ }^{1}$, ZHONG-XI REN $^{2}$, PEI-SONG WANG ${ }^{1}$, CHANG SU $^{1}$, WEN-XIN ZHANG ${ }^{1}$, \\ ZENG-GUANG LIU ${ }^{1}$, LING ZHANG ${ }^{3}$, XUE-JIAN ZHAO ${ }^{3}$ and GUANG CHEN ${ }^{1}$ \\ ${ }^{1}$ Department of Thyroid Surgery, The First Hospital of Jilin University, Changchun, Jilin 130021; \\ ${ }^{2}$ Department of Pathophysiology, College of Basic Medical Sciences of Jilin University, Changchun, Jilin 130021; \\ ${ }^{3}$ Department of General Surgery, Liaocheng People's Hospital, Liaocheng, Shandong 252000, P.R. China
}

Received March 26, 2014; Accepted April 22, 2014

DOI: $10.3892 /$ or.2014.3233

\begin{abstract}
It has been shown that overexpression of signal transducer and activator of transcription 3 (Stat3) contribute to the progression and metastasis of various solid tumors and that silencing Stat 3 inhibits tumor growth in several types of cancer. Gene associated with retinoid-IFN-induced mortality 19 (GRIM-19), a Stat3-inhibitory protein, was identified as a potential tumor suppressor associated with growth inhibition and cell apoptosis by targeting the transcription factor Stat3 for inhibition. However, little is known about Stat3 and GRIM-19 roles in the tumor growth of thyroid carcinoma cells. In the present study, we developed a dual expression plasmid that co-expressed Stat3-specific siRNA and GRIM-19 (pSi-Stat3-GRIM-19) and transfected it into SW579 cells (thyroid carcinoma cell line) to evaluate its effects on cell proliferation, cell apoptosis, cell migration and cell invasion in vitro and tumor growth in vivo. Simultaneous expression of pSi-Stat3-GRIM-19 in SW579 cancer cells was found to significantly suppress the proliferation, migration and invasion in vitro and tumor growth in vivo, when compared to the controls either Stat3-specific siRNA or GRIM-19 alone. In conclusion, our data demonstrated that a combined strategy of co-expressed Stat3-specific siRNA and GRIM19 synergistically and more effectively suppressed thyroid tumor growth, and have therapeutic potential for the treatment of thyroid cancer.
\end{abstract}

\section{Introduction}

Thyroid cancer, although a rare disease, is one of the most common endocrine malignancies, and its incidence is rapidly increasing worldwide (1). Despite the considerable develop-

Correspondence to: Professor Guang Chen, Department of Thyroid Surgery, The First Hospital of Jilin University, 71 Qianjin Street, Changchun, Jilin 130021, P.R. China

E-mail: chenguang518@aliyun.com

Key words: co-expression plasmid, gene silencing, Stat3, GRIM-19, thyroid cancer ments in surgical technique and other therapeutic strategies, the survival rate of patients with thyroid cancer remains low due to its rapid progression and metastasis (2). It is critical to find new methods to treat thyroid cancer (3).

Several reports have shown that signal transducers and activators of transcription 3 (Stat3), a member of STAT family, play a key role in carcinogenesis by promoting cell proliferation, differentiation and cell cycle progression, as well as inhibition of apoptosis $(4,5)$. In addition, constitutive activation of Stat 3 and its overexpression have been detected in a wide variety of human tumors, including prostate (6), breast (7), leukemia (8), lung (9), thyroid cancer (10) and squamous cell carcinoma of the head and neck (11). Targets inhibiting the expression of Stat 3 by RNA interferences could inhibit the proliferation and induce the apoptosis of cancer cells in vitro or suppress tumor growth in a mouse model (12-14). These data suggest that STAT3 may be a new target in the therapy of thyroid cancer.

Gene associated with retinoid-IFN-induced mortality 19 (GRIM-19), an IFN/RA-inducible gene product, was recently identified as a potential tumor suppressor that promotes IFN/RA-induced cell death (15). It has been shown that overexpression of GRIM-19 could inhibit cell proliferation and induce apoptosis in human prostate, breast, gastric cancer and renal carcinoma cells (16-19). Moreover, GRIM-19 has been shown to be an inhibitor of signal transducer and activator of transcription 3 (Stat3) by binding to Stat 3 and suppressing its transcriptional activation $(20,21)$. Taken together, these studies indicate that GRIM9 is a potential tumor suppressor (22).

In the present study, the plasmid expressing GRIM-19 and Stat3-specific short hairpin RNA (p-Si-Stat3-GRIM-19) was transfected into SW579 cells to examine the effect of co-expression of GRIM-19 and Stat3-specific short hairpin RNA on cell proliferation, cell apoptosis, cell migration and cell invasion in a human thyroid carcinoma cell line (SW579 cells) in vitro and on tumor growth in a thyroid cancer xenograft in vivo.

\section{Materials and methods}

Plasmid and cell. Plasmid pSilencerTMneo3.1-H1-Stat3siRNA (pSi-Stat3), plasmid pGCsilencerTMneo3.1-H1- 
scramble (pSi-Scramble), pcDNA3.1-GRIM-19 (pGRIM-19) and Co-expression plasmid pcDNA3.1-GRIM-19-Si-Stat3 (pSi-Stat3-GRIM-19) were granted for the Prostate Diseases Prevention and Treatment Research Center and the Department of Pathophysiology, School of Basic Medicine, Jilin University (Changchun, China). The human thyroid carcinoma cell line, SW579, was obtained from the Cell Bank of the Chinese Academy of Sciences (Shanghai, China).

Cell culture and transfection. The human thyroid carcinoma cell line SW579 was grown in L-15 (HyClone, Logan, UT, USA) with $10 \%$ (v/v) fetal bovine serum (FBS; HyClone). SW579 cells were transfected with various plasmids using the Lipofectamine 2000 reagent (Invitrogen, Carlsbad, CA, USA) according to the manufacturer's instructions for an additional 48-72 $\mathrm{h}$ before analysis of mRNA and protein levels, cell apoptosis and cell proliferation.

Semi-quantitative reverse transcription-PCR (RT-PCR). The mRNA expression levels of Stat3, GRIM-19 and related genes were examined using semi-quantitative RT-PCR. Cells transfected with pSi-Stat3, pGRIM-19 or pSi-Stat3GRIM-19 plasmids were collected after $48 \mathrm{~h}$. Total RNA was extracted using the TRIzol reagent (Invitrogen). Reverse transcription was performed with $5 \mu \mathrm{g}$ of total RNA purified after DNAse I treatment using a commercially available RT-PCR kit (Takara, Dalian, China), based on the manufacturer's instructions. GAPDH was used as control. Stat 3 sense, 5'-GAGTCAGGCACTGTGGG-3' and antisense, 5'-CGGTCGGTTTCTGCCTGTA-3'; GRIM-19 sense, 5'-TTGCCAGTTGTGGTGATC-3' and antisense, 5'-AGAC CCAGAAGGAGCCGC-3'; GAPDH sense, 5'-CCTTCATTG ACCTCAACTA-3' and antisense, 5'-GGAAGGCCATGCC AGTGAGC-3'.

Western blot analysis. Antibodies against Stat3, p-Stat3, survivin, Bcl-2, GRIM-19, matrix metalloproteinase-2 (MMP-2), MMP-9 and $\beta$-actin were obtained from Santa Cruz Biotechnology (Santa Cruz, CA, USA). Anti-rabbit or anti-mouse secondary horseradish peroxidase-conjugated were bought from Amersham Biosciences (Uppsala, Sweden). For western blot analyses, cells were harvested at $48 \mathrm{~h}$ after transfection and lysed with lysis buffer (Takara, Dalian, China). After centrifugation at $15,000 \times \mathrm{g}$ for $30 \mathrm{~min}$, the supernatants were analyzed for protein content using Bradford reagent (Bio-Rad Laboratories, Hercules, CA, USA). After boiling at $100^{\circ} \mathrm{C}$ for $10 \mathrm{~min}$, a total of $40 \mathrm{mg}$ of protein mixed with SDS-PAGE buffer were loaded onto 8-12\% SDS-PAGE gel for electrophoresis. The proteins were separated and then transferred onto polyvinylidene difluoride membranes (Millipore Corp., Bedford, MA, USA). The membranes were blocked in $5 \%$ non-fat milk at $37^{\circ} \mathrm{C}$ for $2 \mathrm{~h}$ and were incubated with the primary antibodies at $4^{\circ} \mathrm{C}$ overnight. After washing three times with TBST buffer, the membranes were incubated with HRP-labeled anti-rabbit IgG or HRP-labeled anti-mouse IgG secondary antibody at $37^{\circ} \mathrm{C}$ for $2 \mathrm{~h}$. The membranes were subsequently washed thoroughly with TBST buffer. The antibody-bound bands were visualized using ECL reagents (ECL; Amersham, GE Healthcare, Velizy-Villacoublay, France) to detect protein. The absorbances of the positive bands in the analysis were measured by densitometry using a GIS Analysis System (Tannon, Shanghai, China).

Measurement of SW579 cell viability. To measure the effect of plasmid pSi-Stat3, pGRIM-19 or co-expression plasmids pSi-Stat3-GRIM-19 on cell proliferation, 3-(4,5-dimethylthiazol-2-yl)-2,5-diphenyltetrazolium bromide (MTT) assay was used. The cell density of SW579 cells was adjusted to $5 \times 10^{4} / \mathrm{ml}$, and added to a 96 -well plate (100 $\mu \mathrm{l} /$ well). In the blank controls, $100 \mu \mathrm{l}$ of medium alone was added. At $24 \mathrm{~h}$ after culture, cells were transfected with different plasmids. At $48 \mathrm{~h}$ after culture, $20 \mu \mathrm{l}$ of MTT $(5 \mathrm{mg} / \mathrm{ml})$ was added to each well followed by incubation for $48 \mathrm{~h}$ at $37^{\circ} \mathrm{C}$. Then, centrifugation was performed at $2,000 \mathrm{x}$ g for $10 \mathrm{~min}$. The supernatant was removed, and $200 \mu \mathrm{l}$ of dimethyl sulfoxide (DMSO) was added to each well followed by shaking for $10 \mathrm{~min}$. Absorbance was measured at $570 \mathrm{~nm}$ with a microplate reader (Molecular Devices Corp., Sunnyvale, CA, USA) and growth inhibition was calculated. The mean proliferation of cells without any treatment was expressed as $100 \%$.

Detection of apoptosis. SW579 cells were cultured in 6-well plates in L-15 containing 10\% FBS medium and were treated with plasmid pSi-Stat3, pGRIM-19 or co-expression plasmid pSi-Stat3-GRIM-19 for $48 \mathrm{~h}$, respectively. The coverslips were washed three times with phosphate-buffered saline (PBS; pH 7.2) and single cell suspensions were fixed in $1 \%$ PBS. Cells were stained with $100 \mu \mathrm{g} / \mathrm{ml}$ acridine orange (AO) and $100 \mu \mathrm{g} / \mathrm{ml}$ ethidium bromide (EB) for $1 \mathrm{~min}$. Then, cells were observed under a fluorescence microscope. At least 200 cells were counted and the percentage of apoptotic cells was calculated.

In addition, in the present study, we also detected survivin and $\mathrm{Bcl}-2$ protein expression by western blotting as an additional indicator of apoptosis.

Wound healing assay. To assess the effect of pSi-Stat3, pGRIM-19 or co-expression plasmid pSi-Stat3-GRIM-19 on cell migration, wound healing assay was performed. SW579 cells $\left(1 \times 10^{5}\right)$ were plated in 12-well plates in complete growth medium. After $24 \mathrm{~h}$ of growth, a scratch was made through the confluent cell monolayer, and then the cells were treated with the indicated plasmid in $3 \mathrm{ml}$ of complete medium. At $48 \mathrm{~h}$ post treatment, cells were stained with hematoxylin and eosin (H\&E). Cells invading the wound line were observed under an inverted phase-contrast microscope (Leica DMR, Wetzlar, Germany).

Cell invasion assay. Cell invasion was determined using Transwell chambers. Transwell filters in 6-well plates were coated with Matrigel, hydrated for $\sim 2 \mathrm{~h}$ in the tissue culture incubator with $500 \mu \mathrm{l}$ serum-free culture media in the bottom and $500 \mu \mathrm{l}$ in the top of the chamber. After hydration of the Matrigel, $5 \times 10^{5}$ SW579 cells were plated in $500 \mu \mathrm{l}$ serum-free L-15 medium on the top chamber, while $2 \mathrm{ml} \mathrm{L}-15$ medium containing $10 \%$ FCS were placed in the lower chambers. Indicated plasmids were added to the upper chambers, respectively. Cells without any treatment were used as control. After $48 \mathrm{~h}$ of incubation, the filters were removed, washed three times with PBS and then fixed in $10 \%$ formalin for $15 \mathrm{~min}$. After fixing at room 
temperature, the chambers were rinsed in PBS and stained with $0.2 \%$ crystal violet staining solution for $30 \mathrm{~min}$. After washing the chambers using PBS, the cells at the top of the Matrigel membrane were carefully removed by a number of cotton swabs. At this time, all cells that remain are the ones that have invaded to the bottom side of the membrane. Cell invasion was observed with an immunofluorescence microscope by counting the cells that had invaded into the bottom of the Cell Culture Insert. In addition, in the present study, we also detected VEGF, MMP-9 and MMP-2 protein expression by enzyme-linked immunospecific assay (ELISA) or western blotting as an additional indicator of invasion.

ELISA. SW579 cells grown in 24-well plates were transfected with the indicated plasmid for $72 \mathrm{~h}$. Protein levels of vascular endothelial growth factor (VEGF) in the cell supernatant were determined by Human VEGF ELISA kit (Yanyu, Shanghai, China) according to the manufacturer's instructions. Samples were measured in triplicate and were properly diluted to ensure that measured values were within the concentration range of the standard curve.

Tumor growth in vivo. Female BALB/c nude mice (Jilin Institute of Experimental Animals) were inoculated with $2 \times 10^{6}$ cells of SW579 cells s.c. into the right flank of the mice. Tumor size was measured every 2-3 days, and tumor volume calculated as $0.5236 \mathrm{x}$ width ${ }^{2} \mathrm{x}$ length. When tumors grew to an average volume of $75 \mathrm{~mm}^{3}$, mice were randomly divided into five groups $(\mathrm{n}=10)$ and inoculated with $20 \mu \mathrm{g} / 50 \mu \mathrm{l}$ per mouse via i.t. injection of different plasmids. Immediately after injection, tumors were pulsed with an electroporation generator (ECM 830, BTX). Pulses were delivered at a frequency of 1/sec, $150 \mathrm{~V} / \mathrm{cm}$, with a length of $50 \mathrm{~ms}$. Mice were sacrificed on day 36, tumor weight and volume was determined, and the tumor blocks were evaluated by RT-PCR assay. Mock mice received implanted tumor but did not receive any plasmid construct therapy or electroporation.

On the 36th day, the animals were euthanized using chloroform and their spleen tissues were collected and cultured for a splenocyte surveillance study. Spleens from treated mice were collected and single-cell spleen suspensions were pooled in serum-free L-15 by filtering the suspension through a sieve mesh with the aid of a glass homogenizer to exert gentle pressure on the spleen fragments. The detailed assay of splenocyte proliferation was based on one previously described (23).

Statistical analysis. Data from at least three independent experiments are expressed as means \pm SD. Statistical comparison of more than two groups was performed using one-way ANOVA followed by a Tukey's post hoc test. Statistical analyses were undertaken using the SPSS ${ }^{\circledR}$ statistical package, version 19.0 (SPSS, Inc., Chicago, IL, USA) and the GraphPad Prism version 5.01 (GraphPad Software, San Diego, CA, USA) for Windows ${ }^{\circledR}$. $\mathrm{P}<0.05$ was considered to indicate a statistically significant difference.

\section{Results}

Effect of pSi-Stat3-GRIM-19 on mRNA and protein expression of Stat3 and GRIM-19. We had three plasmids (pSi-Stat3,
pGRIM-19 and pSi-Stat3-GRIM-19) that are capable of expressing an shRNA that targets the Stat3, tumor suppressor GRIM-19 either alone or in combination. These plasmids were transfected into SW579 cells, a thyroid carcinoma cell line, and their expression was determined using western blot analysis and RT-PCR analyses. It was found that Stat 3 expression on mRNA level and protein level significantly decreased after transfection of expression vectors pGRIM-19, pSi-Stat3 and pGRIM-19-Si-Stat3, and that GRIM-19 expression on mRNA level and protein level was significantly upregulated after transfection with pGRIM-19, pGRIM-19-Si-Stat3 compared to those that received Mock or pSi-Scramble via transfection (Fig. 1).

Effect ofpSi-Stat3-GRIM-19 on cell proliferation and apoptosis in SW579 cells. To investigate if plasmid pSi-Stat3, pGRIM-19 and pSi-Stat3-GRIM-19 exert significantly different effects on cell proliferation, MTT assay was performed for $72 \mathrm{~h}$ when SW579 were transfected with individual expression vectors. Cell proliferation in the pSi-Stat3, pGRIM-19 and pSi-Stat3GRIM-19 groups was significantly diminished compared to the Mock and pSi-Scramble groups ( $\mathrm{P}<0.05$; Fig. 2A). Among the SW579 cell groups treated with pSi-Stat3, pGRIM-19 and pSi-Stat3-GRIM-19, the lowest incidence of cell proliferation was observed in the pSi-Stat3-GRIM-19 treatment group. There was no significant difference between the pSi-Stat 3 and the pGRIM-19 group $(\mathrm{P}>0.05)$.

To investigate whether plasmid pSi-Stat3, pGRIM-19 and pSi-Stat3-GRIM-19 could induce apoptosis, we analyzed the apoptosis after treatment with pSi-Stat3, pGRIM-19 and pSiStat3-GRIM-19. It was found that SW579 cells treated with the pSi-Stat3 and the pGRIM-19 group significantly induced cell apoptosis compared to the Mock and pSi-Scramble groups ( $\mathrm{P}<0.05$; Fig. 2B). Treatment with pSi-Stat3-GRIM-19 led to a marked increase in apoptotic cells compared to the pSi-Stat3 and the pGRIM-19 group ( $\mathrm{P}<0.05$; Fig. $2 \mathrm{~B})$.

To determine the potential mechanism of cell growth inhibition in vitro, apoptosis inhibiting genes, survivin and BCL-2, protein expression were detected using western blots. Survivin and BCL-2 protein expression was significantly decreased in pSi-Stat3, pGRIM-19 and pSi-Stat3-GRIM-19 treatment groups, compared to the controls-Mock and pSiScramble groups $(\mathrm{P}<0.05$; Fig. $2 \mathrm{C}$ and $\mathrm{D})$. Furthermore, the group transfected with pSi-Stat3-GRIM-19 showed the most reduced expression (Fig. 2C and D).

Effects of pSi-Stat3-GRIM-19 on cell migration in SW579 cells. To ascertain the inhibitory effect of pSi-Stat3, pGRIM-19 and pSi-Stat3-GRIM-19 on thyroid cancer on cell motility in vitro, wound healing assay was performed to investigate their effects on the migration potential of SW579 cells. A scratch was introduced into confluent monolayers expressing different treatment plasmids, and the time-dependent movement of cells into the injured area was monitored microscopically. Cells in the Mock and pSi-Scramble groups began migrating $8 \mathrm{~h}$ after scratching. There was no significant change between 8 and $12 \mathrm{~h}$. After $48 \mathrm{~h}$, cells in the pSi-Stat3 group, the pGRIM-19 group and the pSi-Stat3-GRIM-19 group migrated significantly less than those in the Mock and the pSi-Scramble groups. Compared to the $\mathrm{pSi}$-Stat 3 or the 
A

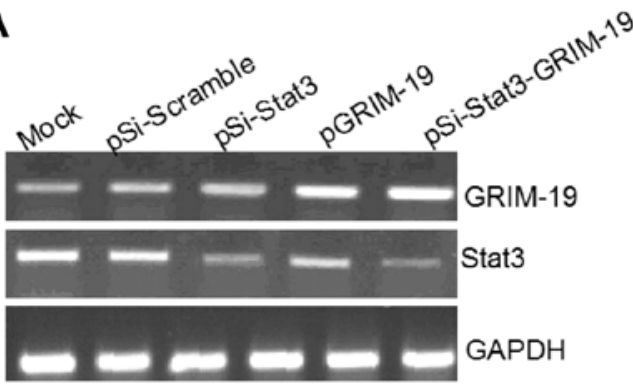

C

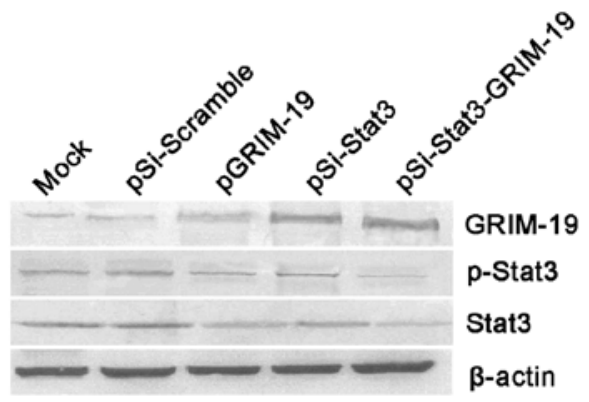

B
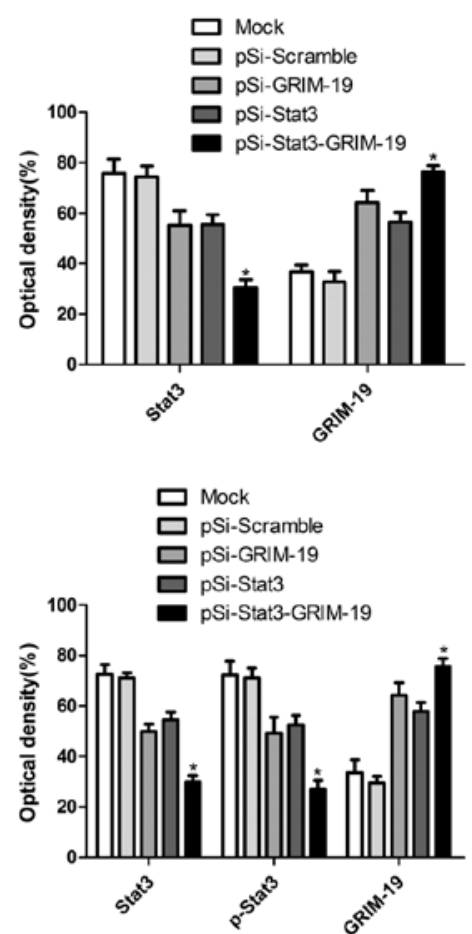

Figure 1. Expression of GRIM-19, Stat3 and p-Stat3 in SW579 cells transfected with various plasmids. (A) RT-PCR analysis of GRIM-19 and Stat3 mRNA; (B) quantification of GRIM-19 and Stat3 mRNA; (C) western blot analyses for Stat3, p-Stat3 and GRIM-19; (D) quantification of Stat3, p-Stat3 and GRIM-19; "P $<0.05$ vs. control.

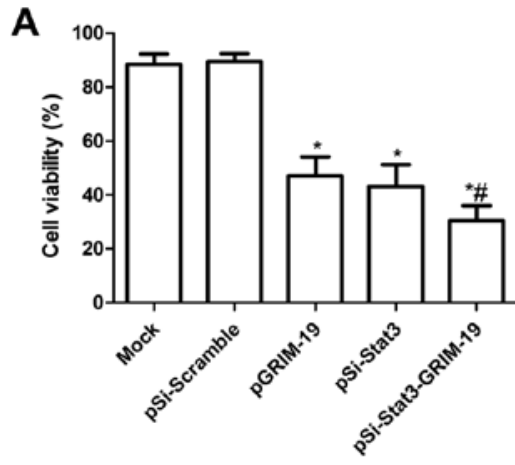

C

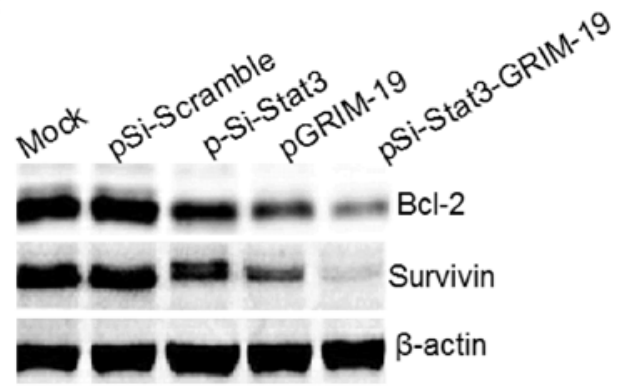

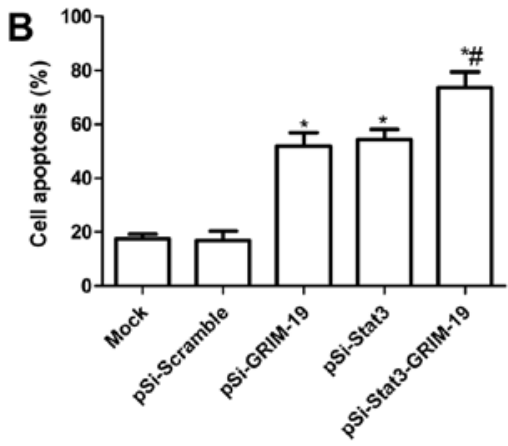
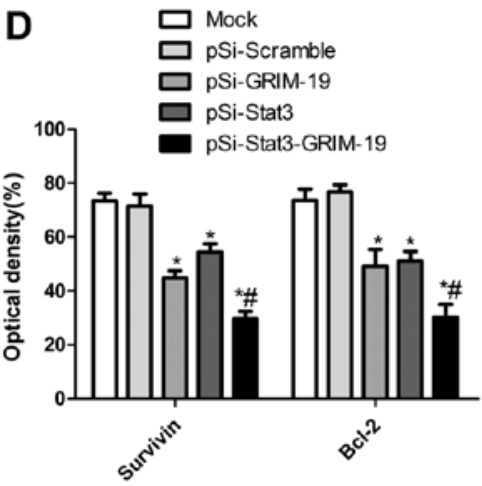

Figure 2. Effects of co-expressed siRNA-Stat3 and GRIM-19 on cell proliferation and apoptosis in SW579 cells. (A) Cell proliferation and (B) cell cycle of SW579 cells was determined $72 \mathrm{~h}$ after treatment with the indicated plasmids. (C) Western blot analysis of survivin and BCL-2 expression. (D) Quantification of survivin and BCL-2 expression; ${ }^{\mathrm{P}}<0.05$ vs. control, ${ }^{\sharp} \mathrm{P}<0.05$ vs. siRNA-Stat 3 alone.

pGRIM-19 groups, the cells in the pSi-Stat3-GRIM-19 group significantly decreased in migration in SW579 cells $(\mathrm{P}<0.05$; Fig. 3A and B).
Effects of pSi-Stat3-GRIM-19 on cell invasion in SW579 cells. The ability of pSi-Stat3, pGRIM-19 and pSi-Stat3-GRIM-19 to reduce the invasiveness of thyroid cancer cells was further 
A

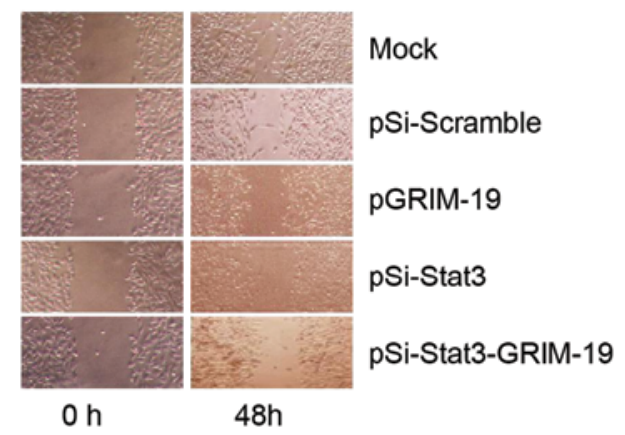

B

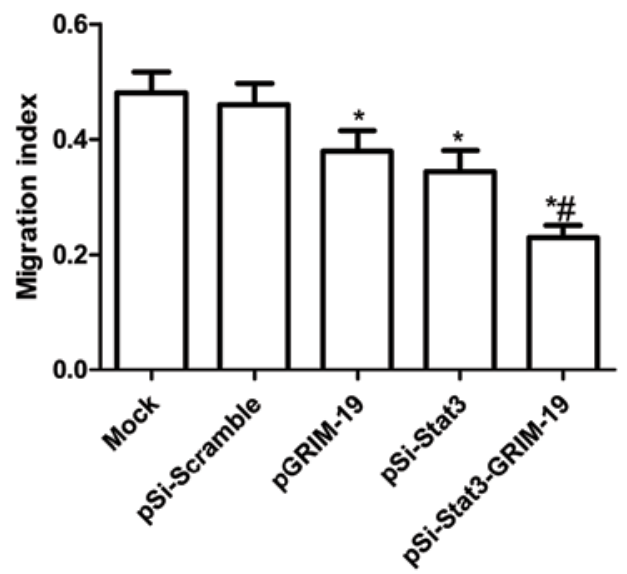

Figure 3. Effects of co-expressed siRNA-Stat3 and GRIM-19 on cell migration in SW579 cells. (A) Cell migration was determined 48 h after treatment with the indicated plasmid; phase micrographs of cells were captured at 0 and $48 \mathrm{~h}$ after monolayer wounding; (B) migration index was counted $48 \mathrm{~h}$ after treatment with the indicated plasmid; ${ }^{*} \mathrm{P}<0.05$ vs. control, ${ }^{\#} \mathrm{P}<0.05$ vs. siRNA-Stat 3 alone.

A

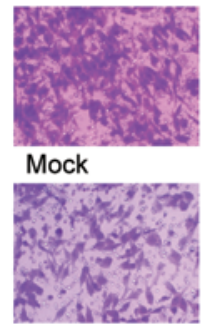

pSi-Stat3

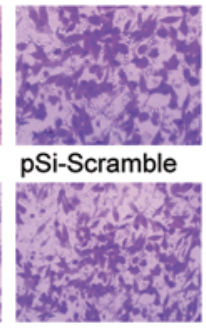

pGRIM-19

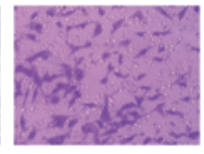

pSi-Stat3-GRIM-19
B

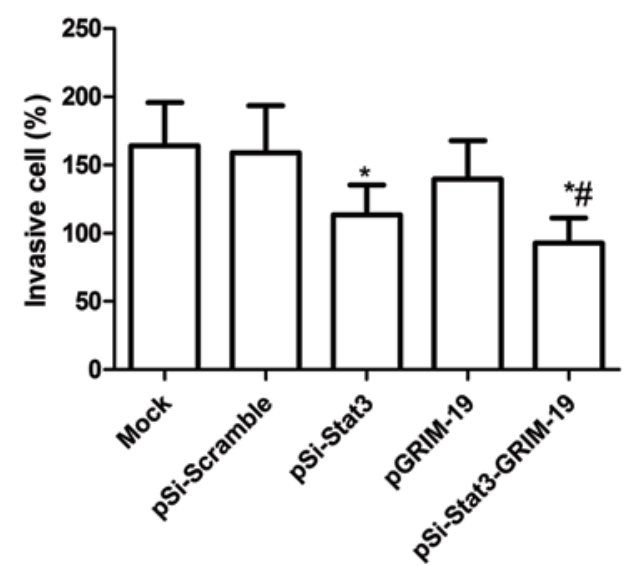

Figure 4. Effects of co-expressed siRNA-Stat3 and GRIM-19 on cell invasion in SW579 cells. (A) Cell invasion was determined by Matrigel 48 h after treatment with the indicated plasmid; (B) the relative number of migration cells $48 \mathrm{~h}$ after treated with indicated plasmid; ${ }^{*} \mathrm{P}<0.05 \mathrm{vs}$. control, ${ }^{\#} \mathrm{P}<0.05 \mathrm{vs}$. siRNA-Stat 3 alone.

investigated by the Transwell system assay. It was found that invasion was also decreased significantly in the pSi-Stat 3 group, the pGRIM-19 group and the pSi-Stat3-GRIM-19 treatment group compared to the Mock and pSi-Scramble groups $(\mathrm{P}<0.05$; Fig. $4 \mathrm{~A}$ and $\mathrm{B})$. Compared to the pSi-Stat 3 or the pGRIM-19 groups, the pSi-Stat3-GRIM-19 treatment group greatly inhibited SW579 cell invasion ( $\mathrm{P}<0.05$; Fig. 4A and B).

pSi-Stat3-GRIM-19 inhibits the invasion-related protein in SW579 cells. To determine the potential mechanism of cell migration inhibition and cell invasion inhibition in vitro, invasion-associated protein expression was examined using ELISA and western blots. As shown in Fig. 5A, ELISA analysis revealed that VEGF excretion in the supernatant from pSi-Stat 3 group, the pGRIM-19 group and the pSiStat3-GRIM-19 group was significantly decreased $(\mathrm{P}<0.05)$, while no obvious changes were observed in the Mock and pSiScramble groups. Western blot analysis displayed a significant decrease in MMP-2, MMP-9 proteins in the pSi-Stat3 group, the pGRIM-19 group and the pSi-Stat3-GRIM-19 group infected SW579 cells compared to Mock and pSi-Scramble groups (Fig. 5B). The pSi-Stat3-GRIM-19 group showed maximally reduced expression compared to either the $\mathrm{pSi}$ Stat3 or pGRIM-19 groups (Fig. 5B). In conclusion, these results suggest that the pSi-Stat3-GRIM-19 inhibitory effect on metastasis of thyroid cancer was at least partially mediated by the downregulation of MMP-9, MMP-2 and VEGF, which may contribute to degradation of the extracellular matrix.

Co-expressed Stat3-shRNA and GRIM-19 synergistically suppress tumor growth in vivo. We next determined if the co-expressed GRIM-19 and siRNA-Stat3 (pSi-Stat3GRIM-19) could synergistically inhibit tumor growth by a xenograft tumor model. Tumor growth was monitored for 36 days. On day 36, animals were sacrificed and final tumor weights and tumor volume were determined. It was found that the tumor weight was significantly lower in various treatment groups than in the Mock and pSi-Scramble groups 
A

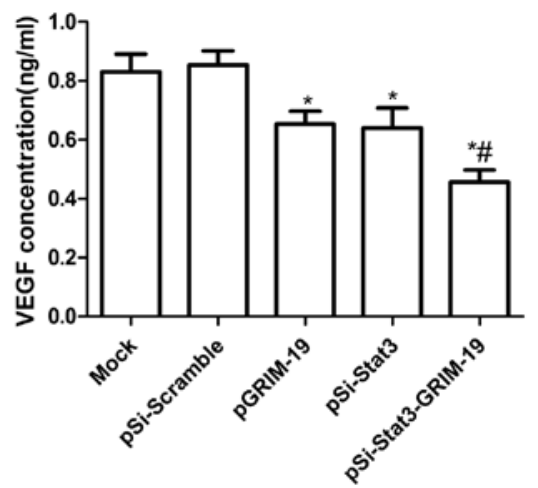

B

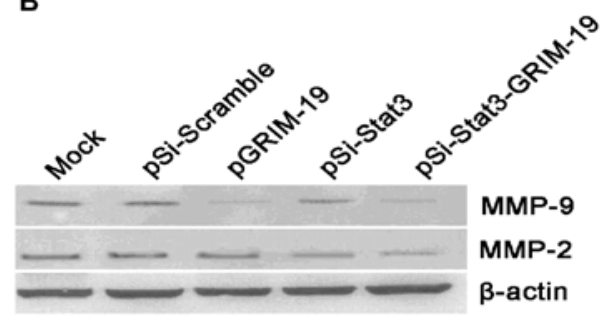

Figure 5. The effect of co-expressed siRNA-Stat3 and GRIM-19 on VEGF, MMP-9 and MMP-2 expression in SW579 cells. (A) The concentration of VEGF was evaluated by ELISA after treatment with the indicated plasmid; (B) western blot analysis was used to assess expression of MMP-2 and MMP-9 using antibodies against MMP-2 and MMP-9. $\beta$-actin was used as an internal control; ${ }^{*} \mathrm{P}<0.05$ vs. control, ${ }^{*} \mathrm{P}<0.05$ vs. siRNA-Stat3 alone.

A

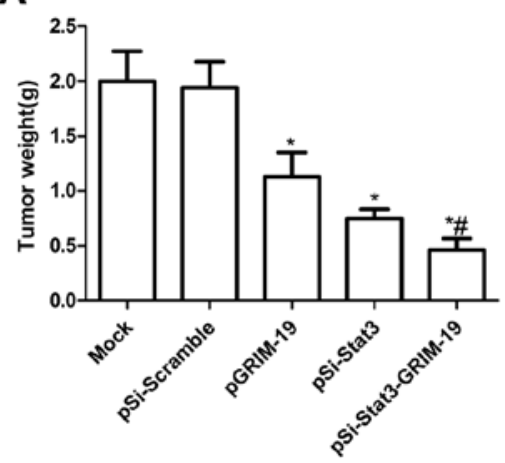

C

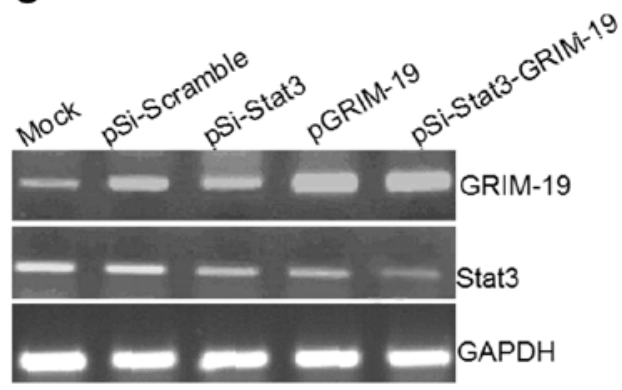

B

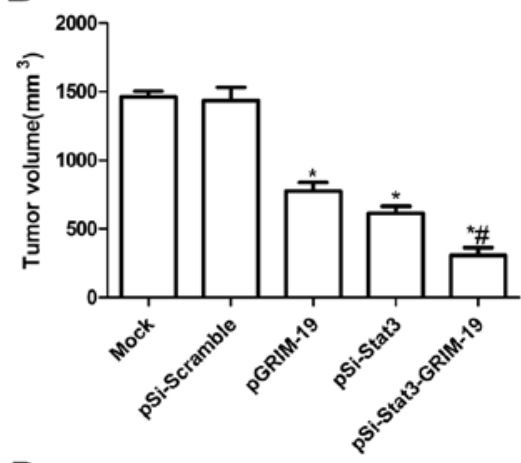

D

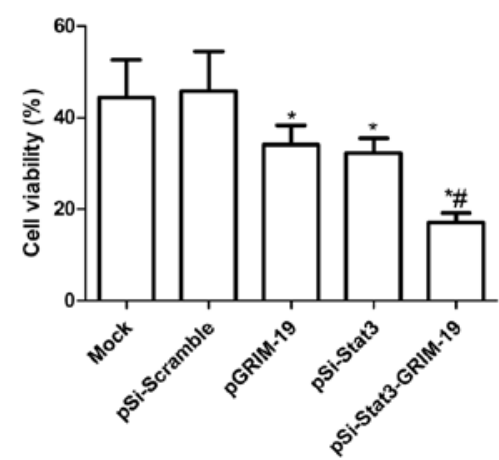

Figure 6. Antitumor activity of co-expressed siRNA-Stat3 and GRIM-19 in BALB/c mice bearing SW579 cells. (A) Tumor weight and (B) tumor volume in treated and untreated mice at 36 days. (C) RT-PCR analyses for Stat3, p-Stat3 and GRIM-19 in tumor tissues after treatment with various plasmids. (D) MTT assay of cell proliferation of splenocytes from mice. Data are expressed as the means $\pm \mathrm{SD}$. ${ }^{*} \mathrm{P}<0.05$ vs. control, ${ }^{*} \mathrm{P}<0.05$ vs. siRNA-Stat 3 alone.

( $\mathrm{P}<0.05$; Fig. 6A). Compared to either the pSi-Stat3 or the pGRIM-19 groups, the pSi-Stat3-GRIM-19 group showed maximally reduced weight. Tumor volume in various treatment groups was significantly $(\mathrm{P}<0.05)$ diminished when compared with the scramble control. Compared with pGRIM-19 and pSi-Stat3, the pSi-Stat3-GRIM-19 group had an obvious effect on tumor growth $(\mathrm{P}<0.01$; Fig. 6B). In addition, in the present study, we also examined the expression of Stat 3 and GRIM-19 in grafted tumor tissues by RT-PCR analysis. It was found that GRIM-19 expression levels were increased in the groups treated with the pGRIM-19 and pSiStat3-GRIM-19 plasmid (Fig. 6C) and that Stat3 expression decreased in the groups treated with pGRIM-19, pSi-Stat3 or pSi-Stat3-GRIM-19 ( $\mathrm{P}<0.05$; Fig. 6C). We also assessed the efficacy of co-expressed GRIM-19 and siRNA-Stat3 (pSi-Stat3-GRIM-19) in modulating splenocyte proliferation using MTT assay. As shown in Fig. 6D, the inhibitory rates of pSi-Stat3, pGRIM-19 and pSi-Stat3-GRIM-19 significantly increased compared to the Mock group and $\mathrm{pSi}$-Scramble group $(\mathrm{P}<0.01)$. The inhibitory rates of the pSi-Stat3GRIM-19 group were higher than those of the pSi-Stat3 and the pGRIM-19 group. These results suggested that co-expressed GRIM-19 and siRNA-Stat3 synergistically inhibited tumor growth in vivo. 


\section{Discussion}

It has been shown that abnormal apoptosis plays an important role in the development and progression of cancer (24) and therefore, controlling the apoptosis of cancer cells has important biological and clinical significance $(25,26)$. Studies have shown Stat3 induced activation via tyrosyl phosphorylation by larger number of different oncogenic events. A large number of studies showed that activated Stat 3 could improve cell proliferation and inhibit cell apoptosis (27-31). In addition, aberrantly active Stat 3 promotes tumor cell growth and survival via an incessant induction of pro-growth genes, such as cyclin D1, c-Myc, survivin, Bcl-xL, Bcl-2, Mcl-1, VEGF and MMP-2 and MMP-9 (27-32), whose products promote tumor cell proliferation, cell cycle progression, metastasis, as well as inhibit apoptosis. Therefore, downregulation of Stat 3 expression could inhibit the proliferation and induce apoptosis. In the present study, our results showed for the first time that downregulation of Stat 3 by RNA interference in thyroid cancer cells significantly suppressed the proliferation, migration and invasion and induced cell apoptosis in vitro, and inhibited tumor growth in vivo. These results were agreement with previous results (12-14).

Although downregulation of Stat 3 has been successfully used for the suppression of tumor growth in vitro or in vivo (12-14), the therapeutic efficacy in vivo has not been fully assessed (12). It is well known that RNA interference does not completely block gene expression, especially when the target mRNA is expressed at abnormally high levels (33). Therefore, in the present study, we chose the plasmid coexpressing GRIM-19 and Stat3-specific short hairpin RNA (pSi-Stat3GRIM-19) to inhibit thyroid tumor growth in vitro and in vivo. GRIM-19, a Stat3-inhibitory protein, has been shown to interact exclusively with the transcriptional activating domain of Stat3 and to inhibit autoregulatory Stat 3 driven transcriptional activation $(15,20,21)$. Alchanati et al (19) showed that reduction of GRIM-19 expression results in upregulation of Stat3-regulated genes in these tumors, and restoration of GRIM-19 suppresses the growth-promoting activities of Stat3. GRIM-19 has been identified as a potential tumor suppressor that promotes IFN/ RA-induced cell death (15), and overexpression of GRIM-19 induces apoptosis, inhibit tumor growth in various types of cancer (16-19). Notably, Wen et al (34) demonstrated that co-expressed survivin-shRNA and GRIM-19 can induce the apoptosis of laryngeal cancer cells and inhibit their proliferation, and co-expressed survivin-shRNA and GRIM-19 is more effective than psi-survivin and p-GRIM-19. Zhang et al (16) reported that the co-expressed Stat3-specific shRNA and GRIM-19 synergistically and more effectively suppressed prostate tumor growth and metastases when compared with treatment with either single agent alone, which was in agreement with our results. In the present study, our results also showed that simultaneous expression of Stat3-specific shRNA and GRIM-19 in thyroid cancer cells significantly suppressed the proliferation, migration and invasion in vitro and tumor growth in vivo, when compared to the controls either Stat3specific siRNA or GRIM19 alone. Our results as well as those of others showed that co-expressed Stat3-specific shRNA and GRIM-19 might be a more effective gene therapy for the treatment of various cancers.
Extracellular matrix degradation is a key step in tumor invasion and metastasis, and it is mainly mediated by the secretion of MMP-2 (32), MMP-9 $(35,36)$ and VEGF $(30,37)$. It has been reported that downregulation of MMP-2, MMP-9 and VEGF contributes to inhibition of cancer cell invasion and metastasis $(38,39)$. In the present study, we found that co-expressed Stat3-specific shRNA and GRIM-19 could suppress thyroid cancer cell migration and invasion and inhibit MMP-2, MMP-9 and VEGF expression.

In conclusion, our results demonstrated that simultaneous expression of pSi-Stat3-GRIM-19 in SW579 tumor cells significantly suppressed thyroid cell proliferation, migration and invasion and induced cell apoptosis in vitro, and inhibited thyroid tumor growth in vivo, when compared to the controls either Stat3-specific siRNA or GRIM19 alone. These results suggest that co-expressed Stat3-specific shRNA and GRIM-19 appears to have therapeutic potential for the treatment of thyroid cancer.

\section{Acknowledgements}

The present study was supported by the Science and Technology Research and Innovation Team funded by the Jilin Province (JL2013018).

\section{References}

1. Jeong SY, Kim HW, Lee SW, Ahn BC and Lee J: Salivary gland function 5 years after radioactive iodine ablation in patients with differentiated thyroid cancer: direct comparison of pre- and postablation scintigraphies and their relation to xerostomia symptoms. Thyroid 23: 609-616, 2013.

2. Siironen P, Hagstrom J, Maenpaa HO, et al: Anaplastic and poorly differentiated thyroid carcinoma: therapeutic strategies and treatment outcome of 52 consecutive patients. Oncology 79: 400-408, 2010.

3. Sakorafas GH, Lappas C, Mastoraki A, Kotsilianou O, Makras P and Safioleas M: Extensive and concurrent infiltration of an ectopic intrathoracic thyroid and thyroid gland by papillary thyroid cancer. Am Surg 76: E152-E153, 2010.

4. Darnell JE Jr: STATs and gene regulation. Science 277: 1630-1635, 1997.

5. Lu Y, Fukuyama S, Yoshida R, et al: Loss of SOCS3 gene expression converts STAT3 function from anti-apoptotic to proapoptotic. J Biol Chem 281: 36683-36690, 2006.

6. Mora LB, Buettner R, Seigne J, et al: Constitutive activation of Stat 3 in human prostate tumors and cell lines: direct inhibition of Stat3 signaling induces apoptosis of prostate cancer cells. Cancer Res 62: 6659-6666, 2002.

7. Li L and Shaw PE: Autocrine-mediated activation of STAT3 correlates with cell proliferation in breast carcinoma lines. J Biol Chem 277: 17397-17405, 2002.

8. Epling-Burnette PK, Liu JH, Catlett-Falcone R, et al: Inhibition of STAT3 signaling leads to apoptosis of leukemic large granular lymphocytes and decreased Mcl-1 expression. J Clin Invest 107: 351-362, 2001.

9. Song L, Turkson J, Karras JG, Jove R and Haura EB: Activation of Stat 3 by receptor tyrosine kinases and cytokines regulates survival in human non-small cell carcinoma cells. Oncogene 22: 4150-4165, 2003

10. Kim EJ, Park JI and Nelkin BD: IFI16 is an essential mediator of growth inhibition, but not differentiation, induced by the leukemia inhibitory factor/JAK/STAT pathway in medullary thyroid carcinoma cells. J Biol Chem 280: 4913-4920, 2005.

11. Grandis JR, Drenning SD, Zeng Q, et al: Constitutive activation of Stat3 signaling abrogates apoptosis in squamous cell carcinogenesis in vivo. Proc Natl Acad Sci USA 97: 4227-4232, 2000.

12. Gao L, Zhang L, Hu J, et al: Down-regulation of signal transducer and activator of transcription 3 expression using vector-based small interfering RNAs suppresses growth of human prostate tumor in vivo. Clin Cancer Res 11: 6333-6341, 2005. 
13. Ling $X$ and Arlinghaus RB: Knockdown of STAT3 expression by RNA interference inhibits the induction of breast tumors in immunocompetent mice. Cancer Res 65: 2532-2536, 2005.

14. Lee SO, Lou W, Qureshi KM, Mehraein-Ghomi F, Trump DL and Gao AC: RNA interference targeting Stat3 inhibits growth and induces apoptosis of human prostate cancer cells. Prostate 60: 303-309, 2004

15. Angell JE, Lindner DJ, Shapiro PS, Hofmann ER and Kalvakolanu DV: Identification of GRIM-19, a novel cell death-regulatory gene induced by the interferon- $\beta$ and retinoic acid combination, using a genetic approach. J Biol Chem 275: 33416-33426, 2000.

16. Zhang L, Gao L, Li Y, et al: Effects of plasmid-based Stat3specific short hairpin RNA and GRIM-19 on PC-3M tumor cell growth. Clin Cancer Res 14: 559-568, 2008.

17. Huang Y, Yang M, Yang H and Zeng Z: Upregulation of the GRIM-19 gene suppresses invasion and metastasis of human gastric cancer SGC-7901 cell line. Exp Cell Res 316: 2061-2070, 2010.

18. Huang G, Chen Y, Lu H and Cao X: Coupling mitochondrial respiratory chain to cell death: an essential role of mitochondrial complex I in the interferon- $\beta$ and retinoic acid-induced cancer cell death. Cell Death Differ 14: 327-337, 2007.

19. Alchanati I, Nallar SC, Sun P, et al: A proteomic analysis reveals the loss of expression of the cell death regulatory gene GRIM-19 in human renal cell carcinomas. Oncogene 25: 71387147, 2006.

20. Lufei C, Ma J, Huang G, et al: GRIM-19, a death-regulatory gene product, suppresses Stat3 activity via functional interaction. EMBO J 22: 1325-1335, 2003.

21. Zhang J, Yang J, Roy SK, et al: The cell death regulator GRIM-19 is an inhibitor of signal transducer and activator of transcription 3. Proc Natl Acad Sci USA 100: 9342-9347, 2003.

22. Nallar SC, Kalakonda S, Lindner DJ, et al: Tumor-derived mutations in the gene associated with retinoid interferoninduced mortality (GRIM-19) disrupt its anti-signal transducer and activator of transcription 3 (STAT3) activity and promote oncogenesis. J Biol Chem 288: 7930-7941, 2013.

23. Kumar BN, Rajput S, Dey KK, et al: Celecoxib alleviates tamoxifen-instigated angiogenic effects by ROS-dependent VEGF/VEGFR2 autocrine signaling. BMC Cancer 13: 273, 2013.

24. Hanahan D and Weinberg RA: Hallmarks of cancer: the next generation. Cell 144: 646-674, 2011.

25. Chau BN and Wang JY: Coordinated regulation of life and death by RB. Nat Rev Cancer 3: 130-138, 2003.

26. Panguluri SK, Yeakel C and Kakar SS: PTTG: an important target gene for ovarian cancer therapy. J Ovarian Res 1: 6, 2008.

27. Turkson J: STAT proteins as novel targets for cancer drug discovery. Expert Opin Ther Targets 8: 409-422, 2004.
28. Masuda M, Suzui M, Yasumatu R, et al: Constitutive activation of signal transducers and activators of transcription 3 correlates with cyclin D1 overexpression and may provide a novel prognostic marker in head and neck squamous cell carcinoma. Cancer Res 62: 3351-3355, 2002.

29. Alas $\mathrm{S}$ and Bonavida B: Rituximab inactivates signal transducer and activation of transcription 3 (STAT3) activity in B-nonHodgkin's lymphoma through inhibition of the interleukin 10 autocrine/paracrine loop and results in down-regulation of Bcl-2 and sensitization to cytotoxic drugs. Cancer Res 61: 5137-5144, 2001.

30. Aoki Y, Feldman GM and Tosato G: Inhibition of STAT3 signaling induces apoptosis and decreases survivin expression in primary effusion lymphoma. Blood 101: 1535-1542, 2003.

31. Wei D, Le X, Zheng L, et al: Stat3 activation regulates the expression of vascular endothelial growth factor and human pancreatic cancer angiogenesis and metastasis. Oncogene 22: 319-329, 2003.

32. Xie TX, Wei D, Liu M, et al: Stat3 activation regulates the expression of matrix metalloproteinase-2 and tumor invasion and metastasis. Oncogene 23: 3550-3560, 2004.

33. Elbashir SM, Harborth J, Weber K and Tuschl T: Analysis of gene function in somatic mammalian cells using small interfering RNAs. Methods 26: 199-213, 2002.

34. Wen LJ, Gao LF, Jin CS, et al: Small interfering RNA survivin and GRIM-19 co-expression salmonella plasmid inhibited the growth of laryngeal cancer cells in vitro and in vivo. Int J Clin Exp Pathol 6: 2071-2081, 2013.

35. Farina AR, Tacconelli A, Vacca A, Maroder M, Gulino A and Mackay AR: Transcriptional up-regulation of matrix metalloproteinase-9 expression during spontaneous epithelial to neuroblast phenotype conversion by SK-N-SH neuroblastoma cells, involved in enhanced invasivity, depends upon GT-box and nuclear factor kappaB elements. Cell Growth Differ 10: 353-367, 1999.

36. Bond M, Fabunmi RP, Baker AH and Newby AC: Synergistic upregulation of metalloproteinase -9 by growth factors and inflammatory cytokines: an absolute requirement for transcription factor NF- $\kappa B$. FEBS Lett 435: 29-34, 1998.

37. Yang EV, Kim SJ, Donovan EL, et al: Norepinephrine upregulates VEGF, IL-8, and IL-6 expression in human melanoma tumor cell lines: implications for stress-related enhancement of tumor progression. Brain Behav Immun 23: 267-275, 2009.

38. Braicu EI, Gasimli K, Richter R, et al: Role of serum VEGFA, TIMP2, MMP2 and MMP9 in monitoring response to adjuvant radiochemotherapy in patients with primary cervical cancer results of a companion protocol of the randomized NOGGO-AGO Phase III Clinical Trial. Anticancer Res 34: 385-391, 2014.

39. Lai WW, Hsu SC, Chueh FS, et al: Quercetin inhibits migration and invasion of SAS human oral cancer cells through inhibition of NF- $\kappa$ B and matrix metalloproteinase-2/-9 signaling pathways. Anticancer Res 33: 1941-1950, 2013. 